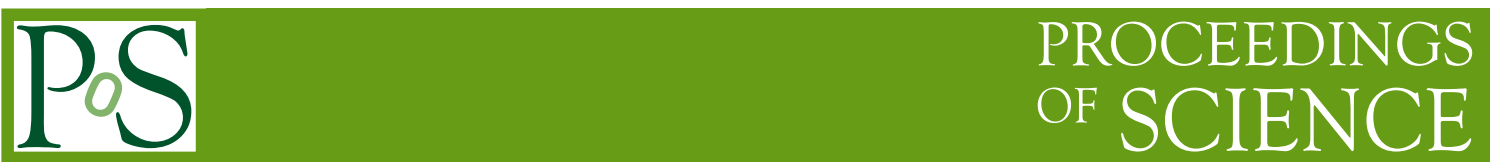

\title{
Charmless b-meson and b-baryon decays at LHCb
}

\author{
Laís Soares Lavra ${ }^{a, 1, *}$ \\ ${ }^{a}$ Université Clermont Auvergne, LPC, CNRS/IN2P3, \\ Clermont-Ferrand, France
}

E-mail: lais.soares.lavra@cern.ch

We present the recent branching fraction measurements of charmless $B^{+} \rightarrow h^{+} h^{\prime+} h^{\prime-}$ decays, where $h$ is a pion or a kaon, using $3 \mathrm{fb}^{-1}$ of pp collisions at $\sqrt{s}=7$ and $8 \mathrm{TeV}$ by the LHCb experiment. The latest measurements of $C P$ asymmetries in charmless baryonic decays are also reported. The updated measurement of the search for $C P$ violation in $\Lambda_{b}^{0} \rightarrow p \pi^{-} \pi^{+} \pi^{-}$decays and the first amplitude analysis of $\Xi_{b}^{-} \rightarrow p K^{-} K^{-}$, which is also the first amplitude analysis of any $b$-baryon decay mode allowing for $C P$ violation effects.

*** The European Physical Society Conference on High Energy Physics (EPS-HEP2021), ***

*** 26-30 July $2021 * * *$

*** Online conference, jointly organized by Universität Hamburg and the research center DESY ***

${ }^{1}$ On behalf of the LHCb collaboration

*Speaker 


\section{Introduction}

Charmless $b$-meson and $b$-baryon decays, due to the predominance of intermediate states, provide an interesting environment to observe $C P$ violation effects. These decays have contribution from both penguin and tree-level processes with amplitudes of similar size and a relative weak phase $\gamma$, which can lead to $C P$ violation effects.

Previous LHCb analysis of $B^{+} \rightarrow h^{+} h^{\prime+} h^{\prime-}$ have revealed large localised $C P$ asymmetries in regions of their phase-space, with results confirmed in amplitude analyses of $B^{+} \rightarrow \pi^{+} K^{+} K^{-}$and $B^{+} \rightarrow \pi^{+} \pi^{+} \pi^{-}$decays[1-4]. The results of fit fractions reported by the amplitude analyses can be converted to quasi-two-body branching fractions, allowing the interpretation of the data. Since the current precision of $B^{+} \rightarrow h^{+} h^{\prime+} h^{-}$branching fractions measurements are not sufficient given the sensitivity of the latest amplitude analyses, improved measurements of these quantities are required. Here, the newest measurements of the relative branching fractions of $B^{+} \rightarrow h^{+} h^{\prime+} h^{\prime}$ are presented.

$C P$ violation phenomenon is a well-established phenomenon in the $B$-meson sector. However, no compelling evidence exists for $C P$ violation in baryonic decays. The large data sample collected by the LHCb experiment enables various studies in this sector to be performed. In these proceedings, two analyses of $b$-baryon decays are presented.

\section{Measurement of the relative branching fractions of $B^{+} \rightarrow h^{+} h^{\prime+} h^{-}$decays}

The relative branching fractions of the decays $B^{+} \rightarrow K^{+} K^{+} K^{-}, K^{+} \pi^{+} \pi^{-}, \pi^{+} K^{+} K^{-}$and $\pi^{+} \pi^{+} \pi^{-}$ are measured using $3 \mathrm{fb}^{-1}$ of Run 1 dataset collected by LHCb [5]. To select signal candidates for each of the four final states, requirements on particle identification variables are applied to reduce the background from misidentified particles. The remaining combinatorial background is then suppressed using a multivariate classifier in which the same requirement is employed for all signal modes. Contribution from $B^{+} \rightarrow \bar{D}^{0} h^{+}$decays that passes through the selection are removed by excluding the corresponding regions in phase space, in addition, tighter particle identification requirements are applied to further remove misidentified charm background components. To extract the signal yields a simultaneous maximum likelihood fit to all signal modes is performed (see Figure 1). The signal yields obtained are $69310 \pm 280$ for $K^{+} K^{+} K^{-}, 94950 \pm 430$ for $K^{+} \pi^{+} \pi^{-}, 5760 \pm$ 140 for $\pi^{+} K^{+} K^{-}$and $25480 \pm 200$ for $\pi^{+} \pi^{+} \pi^{-}$, where the uncertainties are statistical.

The relative branching fractions of two modes are then obtained as the ratio of the signal yields corrected by the efficiency variation over the phase space. The efficiencies are calculated as a function of the Dalitz plot (DP) position, in which corrections are due to differences between data and simulation. Three independent measurements of the branching fractions relative to $B^{+} \rightarrow K^{+} K^{+} K^{-}$ decay are reported,

$$
\begin{gathered}
\mathcal{B}\left(B^{+} \rightarrow \pi^{+} K^{+} K^{-}\right) / \mathcal{B}\left(B^{+} \rightarrow K^{+} K^{+} K^{-}\right)=0.151 \pm 0.004 \text { (stat) } \pm 0.008 \text { (syst), } \\
\mathcal{B}\left(B^{+} \rightarrow K^{+} \pi^{+} \pi^{-}\right) / \mathcal{B}\left(B^{+} \rightarrow K^{+} K^{+} K^{-}\right)=1.703 \pm 0.011 \text { (stat) } \pm 0.022 \text { (syst), } \\
\mathcal{B}\left(B^{+} \rightarrow \pi^{+} \pi^{+} \pi^{-}\right) / \mathcal{B}\left(B^{+} \rightarrow K^{+} K^{+} K^{-}\right)=0.488 \pm 0.005 \text { ( stat) } \pm 0.009 \text { (syst), }
\end{gathered}
$$

where the first uncertainty is statistical and the second systematic, in addition, results for all combinations of one mode relative to another are also reported. All the measurements agree with the current world average and show improvement in all measured ratios' precision. 

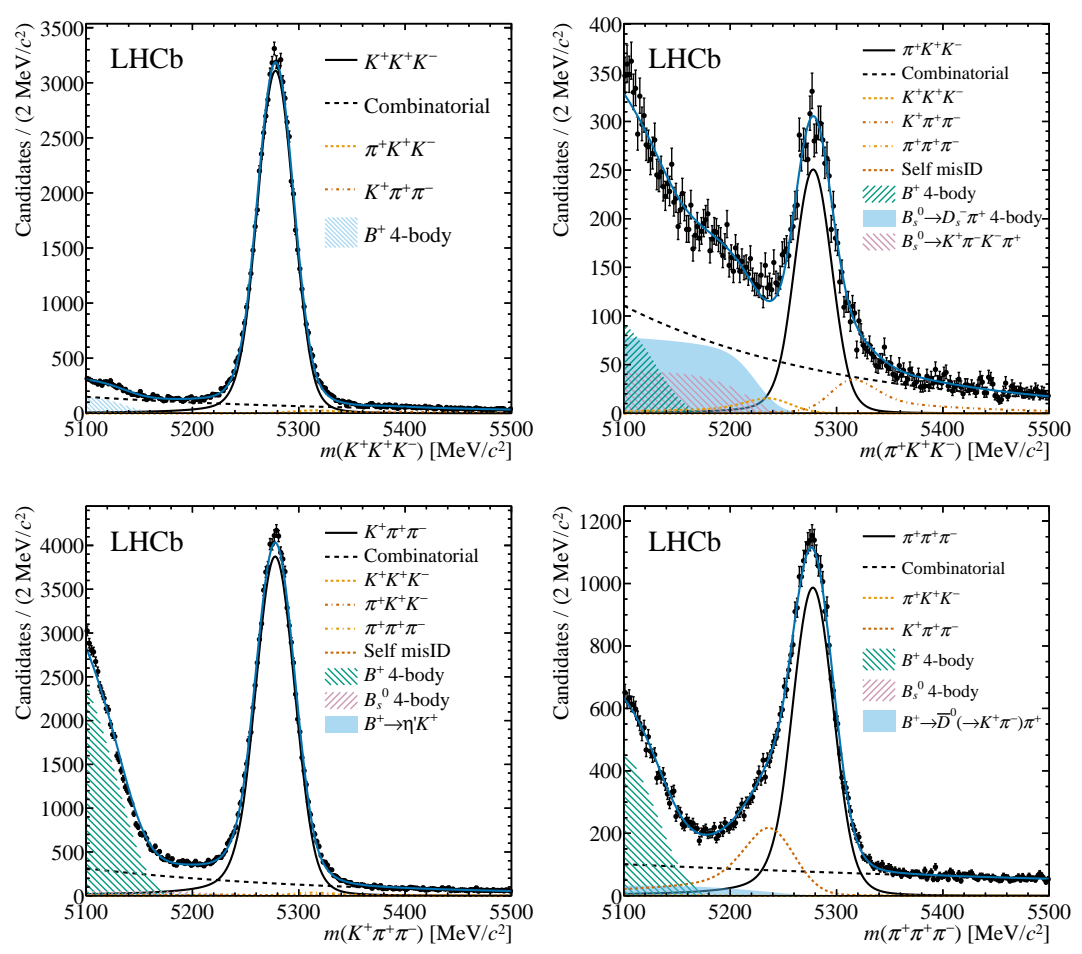

Figure 1: Invariant-mass distribution of the $B^{+} \rightarrow K^{+} K^{+} K^{-}, K^{+} \pi^{+} \pi^{-}, \pi^{+} K^{+} K^{-}$and $\pi^{+} \pi^{+} \pi^{-}$compared to the results of the simultaneous fit.

\section{Search for $C P$ and observation of $P$ violation in $\Lambda_{b}^{0} \rightarrow p \pi^{-} \pi^{+} \pi^{-}$decays}

The LHCb collaboration has previously studied the $\Lambda_{b}^{0} \rightarrow p \pi^{-} \pi^{+} \pi^{-}$and found evidence of localized $C P$ violation with a significance of 3.3 standard deviations [6]. In these proceedings, it is presented the updated measurement reported by $\mathrm{LHCb}$ collaboration using a dataset corresponding to an integrated luminosity of $6.6 \mathrm{fb}^{-1}$ collected from 2011 to 2017 [7]. The signal yields for the $\Lambda_{b}^{0} \rightarrow p \pi^{-} \pi^{+} \pi^{-}$obtained from the mass fit to data is $27600 \pm 200$, which is about four times larger than in the previous analysis. Two independents methods have been chosen for searching $C P$ and $P$ violation in $\Lambda_{b}^{0} \rightarrow p \pi^{-} \pi^{+} \pi^{-}$decays: the triple product asymmetries (TPA), that allows the $C P$ asymmetries to be measured globally and in specific regions of the phase space, and the energy test, designed to look for localized differences in the phase space between two samples.

In the TPA method, $C P$ and $P$ violating observables can be acessed through triple products, defined in terms of the momentum $\vec{p}$ of three final states particles in the centre-of-mass of the $\Lambda_{b}^{0}$ as $C_{\hat{T}} \equiv \vec{p}_{p} \cdot\left(\vec{p}_{\pi_{\text {fat }}} \times \vec{p}_{\pi_{+}}\right)^{12}$. The triple products asymmetries are defined as

$$
A_{\hat{T}}=\frac{N\left(C_{\hat{T}}>0\right)-N\left(C_{\hat{T}}<0\right)}{N\left(C_{\hat{T}}>0\right)+N\left(C_{\hat{T}}<0\right)}, \quad \bar{A}_{\hat{T}}=\frac{\bar{N}\left(\bar{C}_{\hat{T}}>0\right)-\bar{N}\left(\bar{C}_{\hat{T}}<0\right)}{\bar{N}\left(\bar{C}_{\hat{T}}>0\right)+\bar{N}\left(\bar{C}_{\hat{T}}<0\right)}
$$

where $N$ and $\bar{N}$ are the number of $\Lambda_{b}^{0}$ and $\overline{\Lambda_{b}^{0}}$ decays, respectively. Non zero values of TPA do

${ }^{1}$ The $C_{\hat{T}}$ quantity is a $\hat{T}$-odd observable, where $\hat{T}$ is the motion-reversal operator that reverts momentum and helicities.

${ }^{2}$ Hereinafter $\pi_{\text {fast }}^{-}\left(\pi_{\text {slow }}^{-}\right)$refer to the $\pi^{-}$with the highest (lowest) momentum in the $\Lambda_{b}^{0}$ rest frame. 
not mean a sign of $C P$ violation because their values may have influences on final-state interactions (FSI), thus the clean $C P$ and $P$ violation observables are defined as

$$
a_{C P}^{\hat{T}-o d d}=\frac{1}{2}\left(A_{\hat{T}}-\bar{A}_{\hat{T}}\right), a_{P}^{\hat{T}-o d d}=\frac{1}{2}\left(A_{\hat{T}}+\bar{A}_{\hat{T}}\right)
$$

Searches for $C P$ and $P$ violation by measuring $a_{C P}^{\hat{T}-o d d}$ and $a_{P}^{\hat{T}-o d d}$ observables are reported in both integrated and in regions of the phase space. The measured TPA from the fit to the full data set are $a_{C P}^{\hat{T}-o d d}=(-0.7 \pm 0.7 \pm 0.2) \%$ and $a_{P}^{\hat{T}-o d d}=(-4.0 \pm 0.7 \pm 0.2) \%$. The results show no evidence for $C P$ violation, while a parity violation is observed with a significance of 5.5 standard deviations.

Localized measurements were conducted by dividing the phase space into binning schemes $A$ and $B$. In scheme $A$, the asymmetries are measured as a function of the polar and azimuthal angles of $p\left(\Delta^{++}\right)$in the $\Delta^{++}\left(N^{*+}\right)$ rest frame. In scheme $B$, the asymmetries are measured as a function of the angle $|\Phi|$ between the decays planes formed by $p \pi_{\text {fast }}^{-}$and $\pi^{+} \pi_{\text {slow }}^{-}$system. Figure 2 shows the results in bins of the space using the TPA method for the two schemes considered. Each scheme is separated into two subregions $A_{1,2}$ and $B_{1,2}$, where the subscript 1 and 2 are respectively the regions in the phase space dominated by $a_{1}$ resonance and the region with contribution from multiples $N^{*}$ resonances. No evidence for $C P$ violation was found in any of the regions. The measured $C P$ violation with the highest significance was at the level $2.9 \sigma$ in scheme $B_{2}$, and a locally $P$ violation was observed in schemes $A_{1}$ and $B_{1}$ with a significance of 5.1 standard deviations.
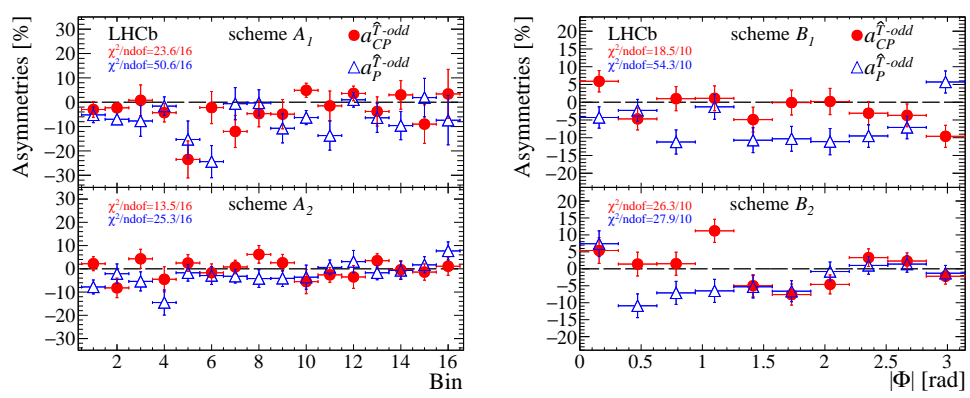

Figure 2: Measured asymmetries for the binning scheme (left) $A_{1}$ and $A_{2}$ and (right) $B_{1}$ and $B_{2}$. The error bars represent the sum in quadrature of the statistical and systematic uncertainties. The $\chi^{2}$ per ndof is calculated with respect to the null hypothesis and includes statistical and systematic uncertainties.

The analysis also searches for $C P$ and $P$ violations using the unbinned energy test technique. The method uses a test statistic, $T$, to calculate localized differences in the phase space between two samples,

$$
T \equiv \frac{1}{2 n(n-1)} \sum_{i \neq j}^{n} \psi_{i j}+\frac{1}{2 \bar{n}(\bar{n}-1)} \sum_{i \neq j}^{\bar{n}} \psi_{i j}-\frac{1}{n \bar{n}} \sum_{i=1}^{n} \sum_{j=1}^{\bar{n}} \psi_{i j}
$$

Here it sums over $n(\bar{n})$ candidates in the first (second) sample, comparing the average-weighted distance of events in phase-space through a metric function $\psi_{i j}=e^{-d_{i j}^{2} / 2 \delta^{2}}$, where $d_{i j}$ is the distance in phase space between the $i$ and $j$ events and $\delta$ is a free parameter that determines the region where the distribution for $\Lambda_{b}^{0}$ and $\bar{\Lambda}_{b}^{0}$ are being compared.

Following the same strategy as TPA, the energy test is performed by dividing the phase space into four subsamples, defined using the triple products. Then, three tests are performed by 
comparing the combination of these four subsamples. For each test, three different values of the parameter $\delta$ are considered. The results are summarized in Table 1 and reported in $p$-values under the hypothesis of $C P$ conserving. All results are compatible with the no $C P$ violation hypothesis. In addition, a local parity violation is observed with a significance of $5.3 \sigma$ for the two smaller distance scale probed.

\begin{tabular}{l|ccc} 
Distance scale $\delta$ & $1.6 \mathrm{GeV}^{2} / c^{4}$ & $2.7 \mathrm{GeV}^{2} / c^{4}$ & $13 \mathrm{GeV}^{2} / c^{4}$ \\
\hline$p$-value $(C P$ conservation, $P$ even $)$ & $3.1 \times 10^{-2}$ & $2.7 \times 10^{-3}$ & $1.3 \times 10^{-2}$ \\
$p$-value $(C P$ conservation, $P$ odd $)$ & $1.5 \times 10^{-1}$ & $6.9 \times 10^{-2}$ & $6.5 \times 10^{-2}$ \\
$p$-value $(P$ conservation) & $1.3 \times 10^{-7}$ & $4.0 \times 10^{-7}$ & $1.6 \times 10^{-1}$
\end{tabular}

Table 1: The $p$-values from the energy test for different distance scales and test configurations.

The parity violation is observed in this decay in both methods, the TPA and the energy test, and the evidence of $C P$ violation previously reported has not been confirmed.

\section{Search for $C P$ violation in $\Xi_{b}^{-} \rightarrow p K^{-} K^{-}$decays}

The amplitude analysis of $\Xi_{b}^{-} \rightarrow p K^{-} K^{-}$decays is performed for the first time, using $3 \mathrm{fb}^{-1}$ of data collected by the LHCb detector in 2011 and 2012 and $2 \mathrm{fb}^{-1}$ collected in 2015 and 2016 [8]. This is the first amplitude analysis of any $b$-baryon decay accounting for $C P$-violation and also presents the search for the previously unobserved $\Omega_{b}^{-} \rightarrow p K^{-} K^{-}$decays.

After the selection of $\Xi_{b}^{-}$and $\Omega_{b}^{-}$candidates, fits to the $p K^{-} K^{-}$invariant mass distribution are performed to obtain the signal yields (see Figure 3). The $\Xi_{b}^{-} \rightarrow p K^{-} K^{-}$yields are $193 \pm 21$ using $3 \mathrm{fb}^{-1}$ and $297 \pm 23$ using $2 \mathrm{fb}^{-1}$ of data. No significant signal of $\Omega_{b}^{-} \rightarrow p K^{-} K^{-}$was found.
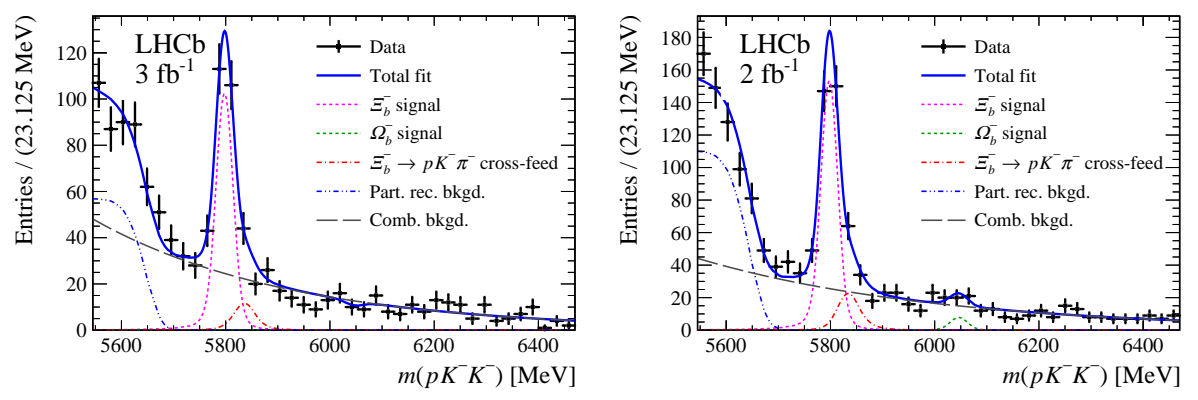

Figure 3: Distributions of $p K^{-} K^{-}$invariant mass for $\Xi_{b}^{-}$and $\Omega_{b}^{-}$candidates in (left) using $2 \mathrm{fb}^{-1}$ and (right) $3 \mathrm{fb}^{-1}$ of data with results of the unbinned extended maximum-likelihood fits superimposed. The total fit result is shown as the blue solid curve, with individual components shown as indicated in the legend.

The results of the fits are used to set an upper limit on the product of $\Omega_{b}^{-} \rightarrow p K^{-} K^{-}$branching fraction with the fragmentation fraction for $\Omega_{b}^{-}$production, $f_{\Omega_{b}^{-}}$, normalised to the corresponding quantities for $\Xi_{b}^{-} \rightarrow p K^{-} K^{-}$,

$$
\frac{f_{\Omega_{b}^{-}}}{f_{\Xi_{b}^{-}}} \times \frac{\mathcal{B}\left(\Omega_{b}^{-} \rightarrow p K^{-} K^{-}\right)}{\mathcal{B}\left(\Xi_{b}^{-} \rightarrow p K^{-} K^{-}\right)}<62(71) \times 10^{-3} \text { at } 90 \text { (95) \% confidence level. }
$$


The search for $C P$ violation is conducted through a model-dependent amplitude analysis, using data with high signal purity of candidates selected in the signal region $\left(\mathrm{m}\left(\Xi_{b}^{-}\right) \pm 40 \mathrm{MeV}\right)$. Assuming $\Xi_{b}$ is produced with a negligible polarisation, the phase space of $\Xi_{b}^{-} \rightarrow p K^{-} K^{-}$is described using two DP variables. The total PDF used to model the phase space is given by

$$
\mathcal{P}_{\mathrm{tot}}^{Q}(\Omega)=\frac{1}{N_{\mathrm{tot}}}\left[N_{\mathrm{sig}} P_{\mathrm{sig}}^{Q}(\Omega)+N_{\mathrm{comb}} \frac{\left(1-Q A_{\mathrm{comb}}\right)}{2} P_{\mathrm{comb}}(\Omega)+\frac{N_{\mathrm{cf}}}{2} \mathcal{P}_{\mathrm{cf}}(\Omega)\right]
$$

where $Q=+1$ for $\Xi_{b}^{-}$and $Q=-1$ for $\bar{\Xi}_{b}^{+}, \Omega$ is the phase space in terms of DP variables and $N$ are the yields. The first term is the signal PDF defined in terms of the signal efficiency $\epsilon$, and the differential decay density $\Gamma$. The second term accounts for the combinatorial background, modelled using neural networks. And the last term corresponds to the cross-feed background PDF, modelled using simulated samples of $\Xi_{b}^{-} \rightarrow p K^{-} \pi^{-}$decay, where the pion is misidentified as kaon.

The output parameters of the amplitude analysis are obtained in terms of the differential decay density $d \Gamma / d \Omega$, defined using helicity formalism for the decay dynamics and the isobar formalism to sum all intermediates resonance. These are the $C P$ violation parameter, $A_{i}^{C P}$ and the fit fractions $\mathcal{F}_{i}$ associated with each intermediate component $i$ of the fit model defined by

$$
A_{i}^{C P}=\frac{\int_{\Omega}\left(d \Gamma_{i}^{+} / d \Omega-d \Gamma_{i}^{-} / d \Omega\right) d \Omega}{\int_{\Omega}\left(d \Gamma_{i}^{+} / d \Omega+d \Gamma_{i}^{-} / d \Omega\right) d \Omega} \quad \mathcal{F}_{i}=\frac{\int_{\Omega}\left(d \Gamma_{i}^{+} / d \Omega+d \Gamma_{i}^{-} / d \Omega\right) d \Omega}{\int_{\Omega}\left(d \Gamma^{+} / d \Omega+d \Gamma^{-} / d \Omega\right) d \Omega},
$$

The model includes contributions from various $\Lambda^{*}$ and $\Sigma^{*}$ resonances that are well established as well as non-resonances contributions. The former are modelled as Breit-Wigner while the latter as exponential. Six contributions are found to give a good description of the data: $\Sigma(1385), \Lambda(1405)$, $\Lambda(1520), \Lambda(1670), \Sigma(1775)$ and $\Sigma(1915)$ resonances. The $C P$ asymmetry for each contributing component is evaluated, and no significant evidence of $C P$ violation is observed. Using the results of the fit fractions, the measurements of quasi-two-body branching fractions for each intermediate component are

$$
\begin{aligned}
& \mathcal{B}\left(\Xi_{b}^{-} \rightarrow \Omega(1385) K^{-}\right)=(0.26 \pm 0.11 \pm 0.17 \pm 0.10) \times 10^{-6}, \\
& \mathcal{B}\left(\Xi_{b}^{-} \rightarrow \Lambda(1405) K^{-}\right)=(0.19 \pm 0.06 \pm 0.07 \pm 0.07) \times 10^{-6}, \\
& \mathcal{B}\left(\Xi_{b}^{-} \rightarrow \Lambda(1520) K^{-}\right)=(0.76 \pm 0.09 \pm 0.08 \pm 0.30) \times 10^{-6}, \\
& \mathcal{B}\left(\Xi_{b}^{-} \rightarrow \Lambda(1670) K^{-}\right)=(0.45 \pm 0.07 \pm 0.13 \pm 0.18) \times 10^{-6}, \\
& \mathcal{B}\left(\Xi_{b}^{-} \rightarrow \Omega(1775) K^{-}\right)=(0.22 \pm 0.08 \pm 0.09 \pm 0.09) \times 10^{-6}, \\
& \mathcal{B}\left(\Xi_{b}^{-} \rightarrow \Omega(1915) K^{-}\right)=(0.26 \pm 0.09 \pm 0.21 \pm 0.10) \times 10^{-6},
\end{aligned}
$$

where the first uncertainty is statistical, the second systematic, and the third is due to the knowledge of $\mathcal{B}\left(\Xi_{b}^{-} \rightarrow p K^{-} K^{-}\right)$.

\section{Perspectives}

The LHCb experiment has a broad upgrade program to significantly improve its luminosity and achieve larger data samples, providing unprecedented precision. With the upcoming data-taking period scheduled to start in 2022 , the combination of $\mathrm{LHCb}$ results will give access to more decays and increase the sensitivity to the $C P$ violation observables. 


\section{References}

[1] LHCb collaboration, Measurements of CP violation in the three-body phase space of charmless $B^{ \pm}$decays, Phys. Rev. D90 (2014) 112004, arXiv: 1408.5373.

[2] LHCb collaboration, Amplitude Analysis of $B^{ \pm} \rightarrow \pi^{ \pm} K^{+} K^{-}$decays, Phys. Rev. Lett. 123 (2019) 231802, arXiv: 1905.09244.

[3] LHCb collaboration, Amplitude analysis of the $B^{+} \rightarrow \pi^{+} \pi^{+} \pi^{-}$decay, Phys. Rev D101 (2020) 012006, arXiv: 1909.05212.

[4] LHCb collaboration, Observation of Several Sources of CP Violation in $B^{+} \rightarrow \pi^{+} \pi^{+} \pi^{-}$decays, Phys. Rev. Lett. 124 (2020) 031801, arXiv: 1909.05211.

[5] LHCb Collaboration, Measurement of the relative branching fractions of $B^{+} \rightarrow h^{+} h^{\prime+} h^{\prime-}$ decays, Phys. Rev. D102 (2020) 112010, arXiv:2010.11802.

[6] LHCb collaboration, Measurement of matter-antimatter differences in beauty baryon decays, Nature Physics 13 (2017) 391, arXiv: 1609. 05216.

[7] LHCb collaboration, Search for CP violation and observation of P violation in $\Lambda_{b}^{0} \rightarrow p \pi^{-} \pi^{+} \pi^{-}$ decays, Phys. Rev. D102 (2020) 051101, arXiv: 1912 . 10741.

[8] LHCb collaboration, Search for CP violation in $\Xi_{b}^{-} \rightarrow p K^{-} K^{-}$decays, Phys. Rev. D 104, 052010,arXiv:2104.15074. 